Physica 136B (1986) 210-212

North-Holland, Amsterdam

\title{
INTRAMOLECULAR AND INTERMOLECULAR INTERACTIONS IN POLYSTYRENE SOLUTIONS
}

\author{
Robert ULLMAN \\ Research Staff, Ford Motor Co., Dearborn, MI 48121, USA
}

J.S. KING

Department of Nuclear Engineering, University of Michigan, Ann Arbor, MI 48109, USA

\begin{abstract}
A series of small angle neutron scattering experiments on polystyrene solutions was used for measurement of radius of gyration, screening length, and an interaction volume as a function of concentration. The results were compared with predictions from scaling theory. In all cases, experiment and theory are in modest disagreement.
\end{abstract}

\section{Introduction}

Small angle neutron scattering (SANS) measurements of polymer solutions containing some labeled (by deuterium) polymer molecules yields both a single molecule scattering function and a collective scattering function of the system [1, 2]. We have done this for polystyrene in deuterotoluene $[3,4]$ and have compared our results with calculations based on scaling laws [5]. A summary of our findings is presented.

\section{Theoretical background}

Consider a polymer solution containing $N$ polymer molecules, each of which is composed of $n$ monomer units. The intramolecular scattering function $S_{\mathrm{s}}(q)$ is defined as

$S_{\mathrm{s}}(\boldsymbol{q})=\left(1 / \boldsymbol{n}^{2}\right) \sum_{i, j} \exp \left[\mathrm{i} \boldsymbol{q} \cdot \boldsymbol{r}_{i j}\right]$,

where $\boldsymbol{r}_{i j}$ is the vector connecting monomer $i$ to monomer $j$, and $q$ is the wave vector, equal to $(2 \pi / \lambda)\left(k-k_{0}\right), \lambda$, being the neutron wavelength and $\boldsymbol{k}_{0}$ and $\boldsymbol{k}$ unit vectors is the direction of incident and scattered neutron beams. The sum is taken over all monomer units within a single polymer molecule. $S_{\mathrm{T}}(q)$ is the total scattering in the absence of isotopic labeling and is equal to

$$
S_{\mathrm{T}}(\boldsymbol{q})=\left(1 / N n^{2}\right) \sum_{\substack{i, j \\ M, M^{\prime}}} \exp \left[\mathrm{i} \boldsymbol{q} \cdot \boldsymbol{r}_{i j}\left(M, M^{\prime}\right)\right] .
$$

$r_{i j}\left(M, M^{\prime}\right)$ is the vector connecting monomer $i$ on polymer molecule $M$ with monomer $j$ on polymer molecule, $M^{\prime}$. The sum is taken over all monomer units on all polymer molecules. $M$ may be, but need not be equal to $M^{\prime} . S_{\mathrm{T}}(q)$ is normalized so that it is equal to $S_{\mathrm{s}}(q)$ in the limit of high dilution.

It has been shown that the intensity of neutrons scattered elastically from the polymer molecules may be written $[1,2,3]$

$$
\begin{aligned}
& I(q)=K\left[A S_{\mathrm{s}}(q)+B S_{\mathrm{T}}(q)\right], \\
& A=\left(a_{\mathrm{H}}-a_{\mathrm{D}}\right)^{2} N_{\mathrm{a}} M c x(1-x) / M_{0}^{2} m, \\
& B=\left(a_{\mathrm{p}}-a_{\mathrm{s}}^{*}\right)^{2} N_{\mathrm{a}} M c / M_{0}^{2}, \\
& a_{\mathrm{p}}=x a_{\mathrm{D}}+(1-x) a_{\mathrm{H}} .
\end{aligned}
$$

$a_{\mathrm{H}}$ and $a_{\mathrm{D}}$ are scattering lengths of protonated and deuterated monomer units, $a_{\mathrm{s}}^{*}$ is a volume adjusted scattering length of the solvent, $N_{\mathrm{a}}$ is Avogadro's number, $M_{0}$ and $M$ are molecular weights of monomer and polymer, $c$ is the concentration of polymer in $\mathrm{g} / \mathrm{ml}$ and $x$ is the mol 
fraction of polymer which is deuterated. $A$ and $B$ are not functions of $q$, and can be changed by variation of either $a_{\mathrm{s}}^{*}$ or $x$ while $S_{\mathrm{s}}(q)$ and $S_{\mathrm{T}}(q)$ remain the same. Eq. (3a) applies if and only if the degree of polymerization of the protonated and deuterated polymers is the same.

From measurements at two values of $x, S_{\mathrm{s}}(q)$ and $S_{\mathrm{T}}(q)$ may be determined independently. $R_{\mathrm{g}}$, the centroidal radius of gyration, can be obtained from $S_{\mathrm{s}}(q)$ unambiguously, and $\xi$, a screening length, may be calculated from $S_{\mathrm{T}}(q)$ using a simple model. $\xi$ measures an effective wave length of concentration fluctuations, and decreases rapidly with increasing solution concentration.

Intramolecular and intermolecular scattering functions are related, and theoretical analysis by Benoit and Benmouna [6] as well as by Jannink and de Gennes [7] lead to the result

$S_{\mathrm{T}}^{-1}(\boldsymbol{q})=S_{\mathrm{s}}^{-1}(\boldsymbol{q})+\left(N_{\mathrm{a}} M c / M_{0}^{2}\right) v$.

The quantity, $v$, is an interaction volume, which at low concentration is proportional to the second virial coefficient. The validity of $\mathrm{Eq}$. (4) for polymer solutions can be examined by measuring the variation of $S_{\mathrm{T}}^{-1}(q)-S_{\mathrm{s}}^{-1}(q)$ as a function of $q$, or by measuring the slope of a plot of $S_{\mathrm{T}}^{-1}(q)$ versus $S_{\mathrm{s}}^{-1}(q)[4]$.

Scaling theory, when applied to polymer solutions, provides predictions of the concentration dependence of varying experimental parameters. If the radius of gyration varies with molecular weight as $R_{\mathrm{g}} \sim M^{\nu}$, the scaling laws yield [5]

$$
\begin{aligned}
& R_{\mathrm{g}} \sim c^{(1-2 \nu) /(3 \nu-1)}, \\
& \xi \sim c^{-\nu /(3 \nu-1)}, \\
& \pi \sim c^{3 \nu /(3 \nu-1)} .
\end{aligned}
$$

The values of $\nu$ for high molecular weights polymer equals 0.6 according to mean field calculations $[8,9]$ and 0.588 according to renormalization group methods [10]. In our experiment (see below), polymer molecular weights are $10^{5}$, and by comparison with earlier experiments [11], we find $\nu=0.57$. We note also that $S_{\mathrm{T}}^{-1}(0)$ varies as
Table I

Predictions for scaling exponents. The exponent $n$ in $X \sim c^{n}$, where $X=R_{\mathrm{e}}, \xi$ or $v$

\begin{tabular}{llll}
\hline & $R_{\mathrm{g}}$ & $\xi$ & $v$ \\
\hline Mean field & -0.25 & -0.75 & 0.25 \\
Renormalization & -0.230 & -0.770 & 0.309 \\
Einaga-Fujita & -0.197 & -0.0803 & 0.408 \\
$\quad$ experiments & & & \\
\hline
\end{tabular}

$\pi / c$ and therefore, $v$ varies as $c^{(2-3 \nu) /(3 \nu-1)}$. Table I contains a list of predictions for the concentration dependence of $R_{\mathrm{g}}, \xi$ and $v$.

\section{Experimental program}

Small angle neutron scattering measurements on H-polystyrene and D-polystyrene were performed over concentrations varying from $0.02 \mathrm{~g} / \mathrm{mol}$ to bulk polymer. The wave vector ranged in magnitude from 0.007 to 0.07 , the deuterated mol fractions were 0.0, 0.5 and 0.8 . Deuterotoluene was used as solvent, and measurements were made at room temperature. Reliable values of $R_{\mathrm{g}}$ were obtained at all concentrations, reliable values of $\xi$ for $c \leqslant 0.16 \mathrm{~g} / \mathrm{ml}$. Eq. (4) could not be checked for $c$ greater than $0.16 \mathrm{~g} / \mathrm{ml}$ owing to increasing relative errors in $S_{\mathrm{T}}(q)$ with increasing concentration. Eq. (4) does not apply exactly, but is approximately valid in the range $0.04 \leqslant c \leqslant 0.16 \mathrm{~g} / \mathrm{ml}$. Best values of $R_{\mathrm{g}}$, $\xi$ and $v$ obey the equation

$$
\begin{aligned}
& R_{\mathrm{g}}=8.6 \times 10^{3} c^{-0.16}, \\
& \xi=2.89 c^{-0.70}, \\
& v=1.52 c^{0.53} .
\end{aligned}
$$

A comparison of the results in eqs. (6a) through (6c) with the predictions of table I shows qualitative correspondence, but also substantial and meaningful quantitative differences. Other measurements of $\xi$ versus $c$ using materials of higher molecular weight are in good agreement with eq. (6b). This tends to discourage us from ascribing the deviation of the prediction of scaling theory from the results of our experiments to the low molecular weight of the polymers used by us. 


\section{Acknowledgement}

This material is based upon work supported by the National Science Foundation under Grant No. DMR-8217460.

\section{References}

[1] A.Z. Akcasu, G.C. Summerfield, S.N. Jahshan, C.C. Han and C.Y. Kim, J. Polym. Sci., Polym. Phys. Ed. 18 (1980) 863.

[2] H. Benoit, J. Koberstein and L. Leibler, MaKromol. Chem., Suppl. 4 (1981) 85.

[3] J.S. King, W. Boyer, G.D. Wignall and R. Ullman, Macromolecules 18 (1985) 709.
[4] R. Ullman, H. Benoit and J.S. King, submitted to Macromolecules.

[5] See, for example, P.G. de Gennes, Scaling Concepts in Polymer Physics (Cornell Univ. Press, Ithaca, 1979) Chap. 3.

[6] H. Benoit and M. Benmouna, Macromolecules 17 (1984) 535.

[7] G. Jannink and P.G. de Gennes, J. Chem. Phys. 48 (1968) 2260.

[8] P.J. Flory, J. Chem. Phys, 17 (1949) 303.

[9] S.F. Edwards, Proc. Phys. Soc. 85 (1965) 613

[10] J.C. Le Guillou and J. Zinn-Justin, Phys. Rev. Lett. 40 (1979) 99.

[11] Y. Einaga, Y. Miyake and H. Fujita, J. Polymer Sci, Polymer Phys. Ed. 17 (1979) 2103.

[12] P. Wiltzius, H.R. Haller, D. S. Cannell and D.W. Schaefer, Phys. Rev. Lett. 51 (1983) 1183. 